Historic, archived document

Do not assume content reflects current scientific knowledge, policies, or practices. 



\title{
PRELIMINARY WHOLESALE PRICE LIST OF SEEDS.
}

Open 30 days from date. For delivery now or Spring 1897.

\section{PETER HENDERSON \& CO. Šeedsmen and Growers,}

\section{$35 \& 37$ Cortlandt Street,}

NEW YORK.

\begin{abstract}
20. This Price List cancels all prevlous quotations and is subject to change without notice.
TERMS OF SALE exactly the same as in our General Wholesale Catalogue issued 1 st January of each year, i. e., Net, 1 st June, 1897 , or Five per cent. discount for Cash in Ten Days.

Delivery F. O. B. New York. 2 bushel seamless bags extra, $15 \mathrm{c}$. each, except where noted.
\end{abstract}

While we exercise the greatest care to have all seeds pure and reliable, we do not give any wuranty express or implier If the purchaser doe not iccept the Seeds on these conditions they must be returned at once and the money that has been paid for same will be refunded.

\section{PEAS.}

All round Peas are $60 \mathrm{lbs}$, to the bushel. Wrinkled sorts $56 \mathrm{lbs}$, to the bu:hel. Wrinkled Peas are marked thus *.

\section{EARLY AND SECOND EARLY.}

Tel.
Cipher. Per bush. ro bush.
lots.

Henderson's First of All,........idum ...\$2 $75 \$ 20$ The best extra early in cultivation. This no charge in made.

Early Morning Star...............Abutment. 250 Extra Early Market.....................Abandon. . 250 Early Kent. ........................... Abdicate.. 200 Early Hancock.......................... Abender... 200 Alaska... .....................Aaron.... 240

Improved Daniel O'Rourke........... Abash .... 225 Philadelphia Extra Early..............Abate.... \& 10 First and Best.....................Abby $y \ldots \ldots 210$ * American Wonder...............Abel..... 800 *Laxton's Alpha $\quad . . . . . \ldots \ldots$........Abject. ... 40 *Extra Early Premium Gem.....Abog......2 285 Blue Beauty.............................. 75 * McLeau's Little Gem. ................. Absent.... ₹ 75 IIcLean's Blue Peter..................Absorb ... . 290 Tom Thumb......................Abstain... 300 *McLean's Adyancer.............. Absurd ... 2 40

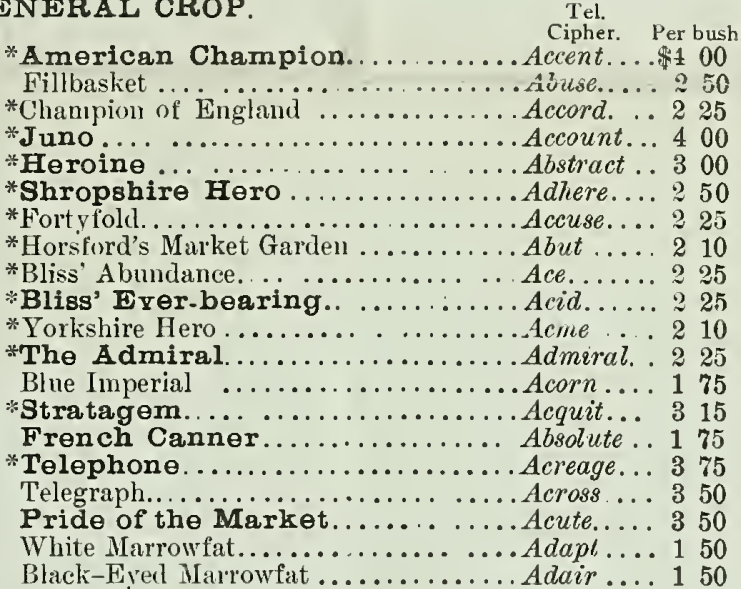

bush. \$3 75

240

210

375

2. 75

240

210

200

210

210

200

210

160

300

160

350

325

340

140 140

\section{BEANS.}

\section{GREEN POD BUSH YARIETIES. T'el. $\begin{gathered}\text { Tipher. Per bush. 'o bush. } \\ \text { Cits. }\end{gathered}$}

Fienderson's New Bush Lima.... Adjunct .. 450 Burpee's Bush Lima.............. Adjure ... 550 Dreer's Bush Lima ................ Adlet..... 500 Henderson's Earijest Red

Valentine.................... Advent ...2 60 Th s earliest round podded variety is sent

$$
\begin{aligned}
& \text { out oniy in } \\
& \text { made. }
\end{aligned}
$$

Early Red Valentine.

Valentine...............Adverb.... 240 Long Yellow Six Weeks................Advice.... 250 Early Mohawk...................Adze..... 250 Refugee, or Thousand to One........Aflux ... 230 Early China.....................Affray.... 2 40 Dwarf Horticultural.............Agony.... 2 50 Best of All.........................Aglow.... 2 40

\section{WAX POD BUSH YARIETIES.}

Challenge Black Wax........... Cipher. Per bush lots. Curries' Rust Proof Wax..........Albatross.. 260 . 60 . 250 Yosemite Mammoth $\mathbf{W} \mathbf{a x} \ldots . . .$. Alegar...6 600 Wardwell's Kidney Wax.............Aldener... 2 60 Keeney's Rustless Wax,............Altruist .. 325 Valentine Wax....................Alwaxit... 350 Golden Wax.....................Alderney.. 250 Cylinder Black Wax............Alderman. 260 Golden Eyed Irax..............Aleak.....2 60 Black Wax or Butter.............. Alert..... 2 50 Improyed Golden Wax..........Alewo..... 260 Flageolet Wax : ...................Algebra... 340 Refugee Wax.................Alight.... 260
Early Golden Cluster Wax........Allowance, 500 King of the Garden Lima.......... Allopath.. 500 Jersey Extra Early Lima.........Alloy..... 550 I.arge White Lima.................Allure.... 450
Dreer's Improved Lima...............Alter.... 450 Old Homestead......................Altro,.... 400 Golden Champion Wax.........Aludel... 500 Horticultural or Speckled Cranberry...Amazon... 350 


\section{General List of Vegetable Seeds.}

\section{ISPARAGUS}

...............Badge....\$0 $60 \$ 5000$

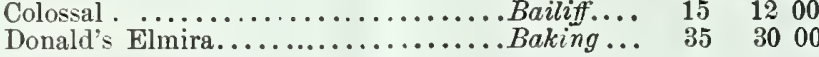

ASPARAGUS ROOT8.

The Palmetto.................Ballad ...75 ${ }^{100}$ Per 50

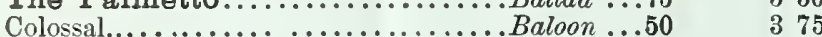

BEANS. (See page 1.)

BEET.

Per lb. soo lbs.

Egyptian Turnip...................Baltic .... $20 \quad 1800$

Eclipse (American grown, selected

stock)........................Bamboo... $20 \quad 1800$

Edmand's Blood Turnip...........Bandle.... 22 2000

Bastian's Blood Turnip..............Bank.... $18 \quad 1600$

Dexing's Improyed Blood

Turn1p .......................Banner... $22 \quad 2000$

Lentz ................................... $22 \quad 2000$

Erlington ..................... Babab... $25 \quad 2200$

Early Blood Turnip................Baptist.... $18 \quad 1600$

Early Flat Bassano ................Barb..... $18 \quad 1600$

Long Smooth Blood Red.................... ${ }_{22} \quad 2000$

CABBAGE.

Henderson's Succession......... Bleach....2 20017500

The Charleston or Large Type

Wakefleld.....................Bleuching. 22520000

Henderson's Autumn King...... Bleat.... 2 20017500

Henderson's Early Spring. ... Blaze. . 7 0060000

Express ........................ Bleary..... 1101000

Henderson's Selected Early Jer-

sey Wakefield. ................Blend..... $150 \quad 14000$

Jersey Wakefield (as generally sold).....Bloom.... $100 \quad \begin{array}{llll}00 & 00\end{array}$

Henderson's Early Summer..... Bluff..... 15014000

Early Summer (as generally sold) ........ Blink ....

Early York .................... Bodkin .. 90

Large Early York.................Bog. .... 9n

Early Winningstadt ...............Bolt ..... $90 \quad 8000$

Fottler's Improved Brunswick........... Booty..... $90 \quad 80 \mathrm{c0}$

Henderson's Selected Late Flat

Dutch........................Botany ... 15014000

Large Late Drumhead .............Bounty... $90 \quad 8000$

Premium Flat Dutch. ..............Brag... . $90 \quad 8000$

Mammoth Rock Red............... Brever ... 2 2520000

Red Dutch............................. Bribe. … 15012500

American Drumhead Saroy ...........Bristle ... 14 4') 12500

CARROT.

Henderson's Intermediate Red ..Cab ..... $35 \quad 3000$

Henderson's Nexw York Market.. Cablet ... 807000

Oxheart or Guerande............Caóal..... 35 3000

Chantenay ........................... $35 \quad 3000$

Early Scarlet Horn ........................ $35 \quad 35 \quad 3000$

Half Long Red (Stump Rooted) . Cadet.... $30 \quad 2800$

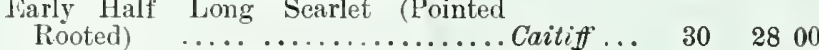

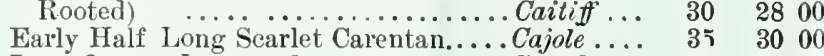

Long Orange Improred .................,lash ... $30 \quad 30 \quad 2800$

Danrers.......................Caldron... $30 \quad 2800$

CAULIFLOWER.

Henderson's Early Snowball

$\$ 2.50$ per $o z$. Packets $\$ 1.50$ per

doz. (these retail at 25c. each)...... Camel ...3500

Extra Early Dwarf Erfurt (per oz. $\$ 2.25$ ). Cameo....30 00

Extra Early Paris .................. Canine ...8 800

Half Early Paris or Nonpareil ........Cannon... 600

Early London ...................... Canoe... 400

Large Late Ägiers (original stock). Canvass .. 700

\section{CELERY.}

Henderson's White Plume........ Capital... $300 \quad 27500$ Golden Self Blanching............ Capivi.... $300 \quad 27500$ Giant Pascal............................... 100 Henderson's New Rose (the finest

flavored sort grown)..............Capon....2 00 Henderson's Golden Drarf. ....Caprice...\$1 25 Henderson's Half Dwarf...........Capsize.... 100
CELER Y.-Continued.

Tel.

Dxarf White .......Carbine... 150

arf White ............Caress..... 125

Boston Market ..................... Carola.... 1 1 25

London Red.............................. 125

Celeriac, or Turnip-rooted Celery.

Large Smooth Prague...........Castor... 150

Erfurt Giant.......................Cavalry... 100

CORN. Sugar.

CAUTION.-Sweet Corn in bulk, even after being thoroughly cured, will often gather moisture and spoil. particula:ly if it has been $E X-$ posed to a low temperature. To prevent this, it should be, taken out of the bag as soon a RUOTATIONS ARE PER MEASURED BUTHEL, SHELLED. All eastern Per ro bush, bush. lots.

Country Gentleman. ..........Censual... 2 $75 \quad 250$

Ne Plus Ultra $\ldots . \ldots \ldots \ldots \ldots \ldots \ldots$. Census.... $250 \quad 25$

The Henderson................. Centaur... 2 $00 \quad 180$

White Cob Cory................Centime... 240225

old Colony.........................Century .. $200 \quad 180$

Extra Early Tom Thumb..................... 1 is 15 (5) 160

Roslyn Hybrid... ...............Chain.... 2 252200

Early Narblehead.................Channel . $200 \quad 180$

Cory Early (Red Cob)... ........ Chapel .. 2 25 200

Early Minnesota ................... Chariot .. $175 \quad 160$

Stabler's Early ................... Charmer. 225200

Crosby's Early .....................Chasm.... 2 $00 \quad 180$

Large Early Eight-Rowed. ..........C hattel... $200 \quad 180$

Early Adams, or Burlington ...........Chemist...2 25 200

Moore's Early Concord............... Cherish...200 180

Hickox Improved ................Ch rub... $175 \quad 160$

Squantum.................... Chess.... \& 25 200

Egyptian ................................ $200 \quad 180$

Stowell' Eyergreen ....................... 175

Triumph ......................Chintz... 1 i5 160

Mammoth, Large, Late............ Choral... $175 \quad 160$

Potter's Excelsior.................. Cinderella. 225200

Perry's Hybrid ..................... Cinder... $225 \quad 250$

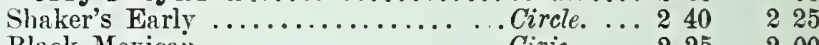

Black Mexican. ...................Civic.. .. 225

200

CORN SALAD OT FETTICUS.

Per lb. $100 \mathrm{lbs}$

Large Leaved................. Cobalt.... $28 \quad 2500$

CUCUMBER

Cool and Crisp ............... Cognate... 50 4000

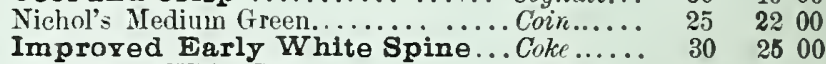

Extra Long White Spine............Combat... 28 25 00

Japanese Climbing........................ $80 \quad$ 7000

Everbearing ... .................Comedian. 30 25 00

Livingston's Evergreen ............Comest.... $25 \quad 2200$

Early Frame....................Cometer.. 25 2200

Early Cluster..................... Comfort.. $25 \quad 2200$

Short Green....................... Commune. $\quad 25 \quad 2200$

Green Prolifie...................... Compass.. $\quad 25 \quad 2200$

Extra Early Green Proliflc, (simi-

lar to the ordinary Green Prolific, but

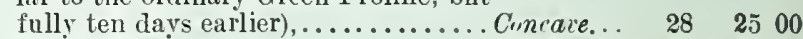

Early Russian......................... Conceited. $\quad 28 \quad 2500$

Long Green .....................Concert... $28 \quad 2500$

Long Green Turkey................ Couch.... $28 \quad 2500$

West India Gherkin, or Burr........... Cone..... 80

EGG PLANT.

New York Improved............... Content... 225

Nex York Improxed Spineless.. Construe.. 250 ENDIVE

Green Curled (winter)...............Copper.... 100

French Moss Curled.......................... 125

Broad Leared Batavian,...................... 100

KALE OT BORECOLE.

Siberian, German Greens or " Sprouts". Cordial... $28 \quad 2500$

Dwarf Green Curled Bcotch. ...Cornet.... 35
3000 
LEEK.

Tel.

Cipher, Per lb. roo lbs.

Large American Flag..............Council...\$0 $80 \$ \$ 00$

Musselburgh............................ 1 2.5

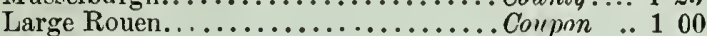

Carentan........................... 140

LETTUCE.

Henderson's New York, w. s..... Court.... 807000 Golden Queen, w. s...................... $100 \quad 9000$ Henderson's Big Boston, w. s. Be-

ware of inferior stocks of this offered

under the name of Large Boston, etc.Courticr... $\quad 90 \quad 8000$

Sunset, w. s........................... $50 \quad 4500$

Early Curled Simpson, w. s....................... 50 50 4500

Black Seөded Simpson, B. s...... Cozey .... $55 \quad 5000$

Salamander, Extra Selected Market

Gardeners' Stock, в. s ............ Crabidge.. 1009000

Salainander, B. s. ........................ $50 \quad 4500$

Yellow Seeded Butter, y. s................ $50 \quad 4500$

Reichner's Forcing, w. s ............ Crampit... $\mathbf{5 5} 5000$

Prive Head, w. s....................... $45 \quad 4000$

Tennis Ball, Black Seeded, B. s................. $50 \quad 4500$

Black Seeded Butter, B. s.................. 5rane .. 4500

Boston Market, w s, . .......... Cranky... 55 5000

Large White Summel Cabbage, w. s ..Craxh.... $\mathbf{5 5} \quad \mathbf{5 0} 00$

Drumbead, or Malta, w. . ..........'('rater.... 511 4500

Hanson, w. s .......................... Crayon... 50 4500

All the Year Round, B. s............ Craze .... so so 4510

Hardy Grcen Winter, w. s ........... Cr dot.... 4.5 40

Deacon, w. s .......................... Creep... 504500

Nex Cos or Celery Lettuce,

Trianon, w. s...................etiner... 70 6500

Paris White Cos, w. s...................... $60 \quad 5500$

MELON, Musk.

Perfected Delmonico............ Craigo.... $60 \quad 5000$

Banquet.................................. . $50 \quad 4000$

New Early Hackensack ............ Oricket... $85 \quad 3000$

Newport . .................. Crick..... 1009000

Fmerald Gem................. Criper.... $35 \quad 3000$

Miller's Cream...................... Crinoxe $\ldots . . \quad 35 \quad 3000$

Delmonico. ............................ $\quad 25 \quad 2200$

Princess .........................

Baltimore. .................. Crisis..... $25 \quad 2200$

Montreal Market.......................... $25 \quad 2200$

Jenny Lind............................. ('. $35 \quad 3000$

Green Citron . ................. Crumy .... $25 \quad 2200$

Nutmeg ............................. 25 2200

Skillman's Netted... .............Croton.... $25 \quad 2200$

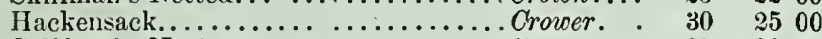

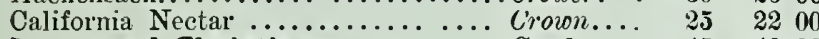

Improved Christiana....................... $45 \quad 4000$

Ward's Nectar................... Crudle... $25 \quad 2200$

Surprise. $\quad \ldots \ldots \ldots \ldots \ldots \ldots \ldots \ldots$. . ruive ... $30 \quad 2500$

Golden Netted Gem ......................... $35 \quad 3000$

MELON, Water.

Cole's Early....................Cumnock . $\quad 50 \quad 4000$

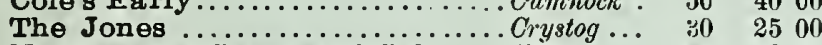

Henderson's Green and Gold ....... Ćubit..... $20 \quad 1800$

Florida Favorite................Cuboia.... $25 \quad 2200$

The Volga. ......................... $20 \quad 1800$

Jordan's Gray Monarch. .......................... $30 \quad 2500$

Mammoth Ironclad .................... $30 \quad 2500$

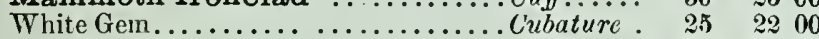

Pride of Georgia. ................. Cudiel .. $25 \quad 2200$

Dixie.......................Cullion.... $25 \quad 2200$

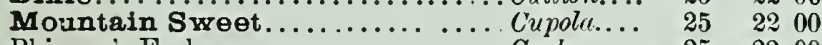

Phinney's Early............................. 2urb.... 25 2200

Scaly Bark ................... Curfew ... $20 \quad 1800$

Kolb's Gem... .................... $20 \quad 1800$

Black Spanish........................... $30 \quad 2500$

Ice Cream (White Seeded).................... 2utlass ... 25 2200

Gypsy...................... Cutter.... $\quad 25 \quad 2200$

Georgia Rattlesnake... ..................... $25 \quad 2200$

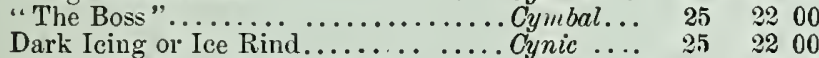

California or Improved Odella...................... $25 \quad 2200$

Light Icing...................... Cyma. .. $25 \quad 2200$

Cuban Queen ......................... $25 \quad 2200$

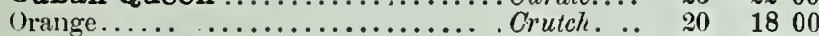

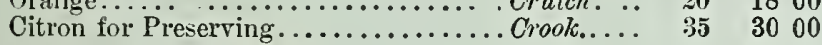

\section{MUSHROOM SPAWN.}

English Dagger....

French
ONION Y' Wethersfield Large Red, Imported......Daze..... $60 \quad 5500$

Yellow Globe Danyers. $\quad . . .$. Debate ... $\quad 656000$

Yellow Globe Danvers, Imported......... Debacle.... 50

Round Yellow Danvers . ........... Decamp... $55 \quad 5000$

White Portugal or Silyer Skin. Deck...... 5514000

White Portugal or Silver Skin, Imptd..Decanter.. 11010000

White Bunch.. .............. Decrease.. 140

Southport White Globe .........Decree.... 15014000

Southport White Globe, Imported.. . . Decretal... 1009000

Large Southport Red Globe...... Defy... 807000

Large Southport Red Globe, Imported... Deface ... $60 \quad 6500$

Southport Yellow Globe ......Delta.... $80 \quad 7000$

Southport Yellow Globc, Imported. ...Delete..... $60 \quad 5500$

Prizetaker ............................. 11010000

Adriatic Barletta................. Derign.... 1 25 11000

Giant Rocca.............................. 80 . 7000

ฆ Mammoth Pompeii, or Red Garganus. Mervish.... $90 \quad 81100$

Neapolitan Marzajola ............ Device ... 1009000

Que日n Early Pickling..............Dero...... 11010000

$\{$ Large White Italın Tripoli...................... 70 7irk 6000

W Large Red Italian Tripoli...................... 908000

Giant White Garganus, or Silrer

King....................Dismiss . .

ONION SETS. Net Cash. Prices on application.

PARSNIP.

Long.Smooth or Hollow Crown.. Docile..... 25

2200

PARSLEY.

Henderson's Emerald ...........Dolphin.

Extra Double Curled.............Domain... 35

PEAS. (See page 1.)

POTATOES. (vet Cash, prices subject to variation.) We deliver all Potatces in to the hands of transportation companies safely packed; after that our responsibility ceases. Purchasers must take all risks from treezing or heating. In comparing our prices on Potatoes, remember we make no charge for barrels or cartage,
liver F. O. B., N. Y, at price quoted.

Henderson's LATE Puritan..... . Kadish

Rochester Rose..................Endem .

Restaurart............. ... Eafing.

Snowdrop................... Eagat. . .

Henderson's Early Puritan .... Eager.

"Bill Nye".................. kagleing.

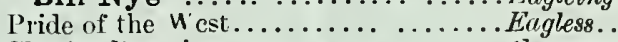

Charles bowning. ............... . . .

Rural New Yorker, No. 2 ....Eanting

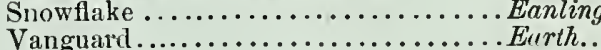

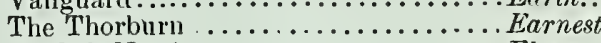

Clarke's No. 1.................Ebony

Beauty of Hebron .. ..............Eeho

Early Maine ................... Eden

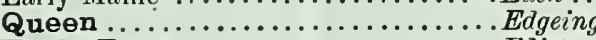

Early Rose................... Edict

Early Ohio ................. Edifice.

Early Sunrise..................Editor

Triumph $\ldots \ldots \ldots \ldots \ldots \ldots \ldots \ldots \ldots$ Efface

Pearl of Sayoy............... Eject.

Empire State..................Elapse

Early Norther................ Earnorth

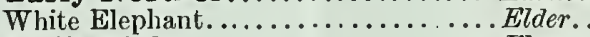

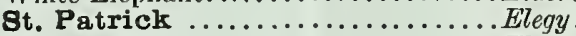

White Star.................. Element

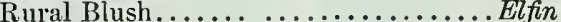

Morning Star .................Elixir....

PUMPKIN.

Calhoun

Large Che

Jumbo

Large Tours or Mammoth ............. Emdire..

RADISH.

Early Round Dark Red.........Encore....\$0 22 2000

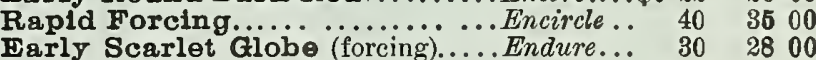


RADISH.-C'ontinned.

Red Forcing Turuip .

Beckert's Chartier

Tel.

Clpher, Per lb, $100 \mathrm{lbs}$

Endorsed.. $\quad 35 \quad 3000$

Enemy... $\quad 28 \quad 2.500$

Early Scarlet Turnip ............Energy.... $\quad 22 \quad 2000$

$\begin{array}{lll}\text { Long Searlet Short-top. } & 28 & 2500\end{array}$

Wood's Early Frame............Engine.... $28 \quad 2500$

French Breakfast .................. Engruve... $30 \quad 2800$

White Tipped Scarlet Turnip... Enquire. $22 \quad 2000$

Large White Summer Turnip......... Enrage $\quad 30 \quad 2800$

Giant White Stuttgart (Summer) ....E Enrap ... $28 \quad 2500$

Yellow or Golden Summer

Turnip...

Enrich . $25 \quad 2200$

Long Brightest Scarlet ......... Enclasp. $\quad 30 \quad 2800$

Long Thite Tienna or Lady Finger..... Entwine.. 30 is 00

Rose China Winter..............Enxiron... $40 \quad 3500$

Round Black Spanish...............Episode... 28 2.5 00

Long Black Spanish .............Em y $\ldots 28 \quad 2500$

SALSIFY, or Oyster Plant.

Improved Mammoth Sandwich

Island..$\ldots \ldots \ldots \ldots \ldots \ldots \ldots \ldots$ Epode.... so $80 \quad 7000$

Large White................... Equip ... 50 $50 \quad 4500$

SPINACH.

Henderson's Norfolk Sayoy

Leaver......................Fabian.... $11 \quad 900$

Round Leaf...........................Fable.... 10 \& 00

Long Standing...................Facile.... $10 \quad 8 \mathrm{C0}$

Large Round Leaf Viroflay............Faculty... $12 \quad 1000$

New Thick Leared................Fugot..... 1? 1000

SQUASH.

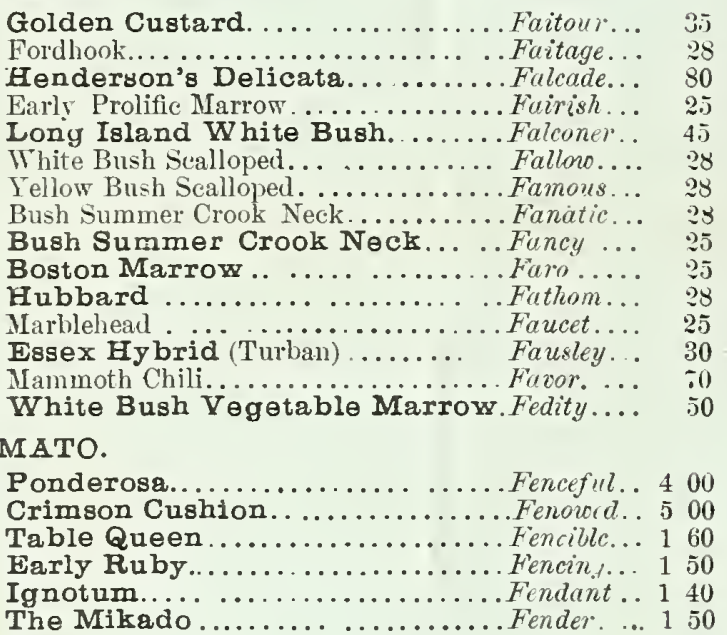

2200

2200

2500

2200

2800

6000
TOMATO.-Continued.

Optimus........................ Ferret ... 125

Stone............................ Fendicul . 150

Fayorite. .....................Ferry . 1 2.

Perfection ...................... Fervid... 125

Acme ................................ 125

Cardinal........................Fickle.... I 20

Paragon .... Fetoon... 130

Beauty ......................Fetch.... 140

Mayflower .............................. 110

Trophy, Finest, Extra selected ..Fiasco.... 150

Essex Hybrid. ............. .....Fibre.... 130

General Grant ................... Fiddle . . 110

Hathayay's Excelsior............. Fidget.... 110

Golden Sunrise ................ Filose..... 140

Pear Shaped. Red.... ............ Finch... 1 is

Yellow Plum ...................... Fiscal ... 1 is

TURNIP.

Early Snowball ...................Gabled. . 35 3000

Red Top Strap Leaf..................Galaxy $\ldots . .630 \quad 3500$

Early White Strap Leaf............. Gale ..... ?8 2400

White Ego....................Gamble.. $30 \quad 250$

Purple Top White Globe.........Gander... $28 \quad 2100$

Early Flat Dutch................Gap..... 28 2400

Yellow Globe.....................Garnet... 30 30,9500

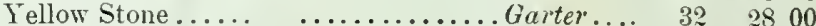

Long White. or Cow Horn ............Gasping... $30 \quad 2500$

Golden Ball....................Gauge ... $30 \quad 2500$

Yellow Aberdeen Purple Top....Gare? ... $25 \quad 2200$

RUTA BAGA, RUSSIAN OOT SWEDISH TURNIP.

Long Island Improyed .. .......Guzette... 32 is 10

Improyed American Purple TopGenera.... $28 \quad 2400$

Laing's Improred ................ Genial ... $25 \quad 2000$

Skirying's Purple Top..........Gentry.... 201800

RAPE, English..................Gimlet... \& $8{ }_{6} 00$

\section{LAWN GRASS.}

The "Henderson" Lawn Grass, A new mixture

Per bush. and composed of the rery finest samples of various grasses which, by actual experiments, we have found best adapted to produce a PERFECT LAWN. Sold either in 1, 2

and 4 quart packages or in bulk, (bags included). . Nutul

400

The "Henderson" Lawn Grass is put up in attractive boxes of new design. and it will be found the most salable mixture on the market. (Full printed instructions of "How to make a Lawn" in each package )

This mixture weighs 20 to 21 lbs, per bushel. Quotations per STANDARD bushel of 14 lbs, are often misleading. If followed in thi instance. it will be seen that this mixture is really at $\$ 2.75$ per bushel of $14 \mathrm{lbs}$.

Io $1 \mathrm{bs}$. at roo $1 \mathrm{~b}$, rates.

\section{SWEET PEAS.}

\section{Those prefixed with an asterisk are Eckfords Large Flowering Varipties.}
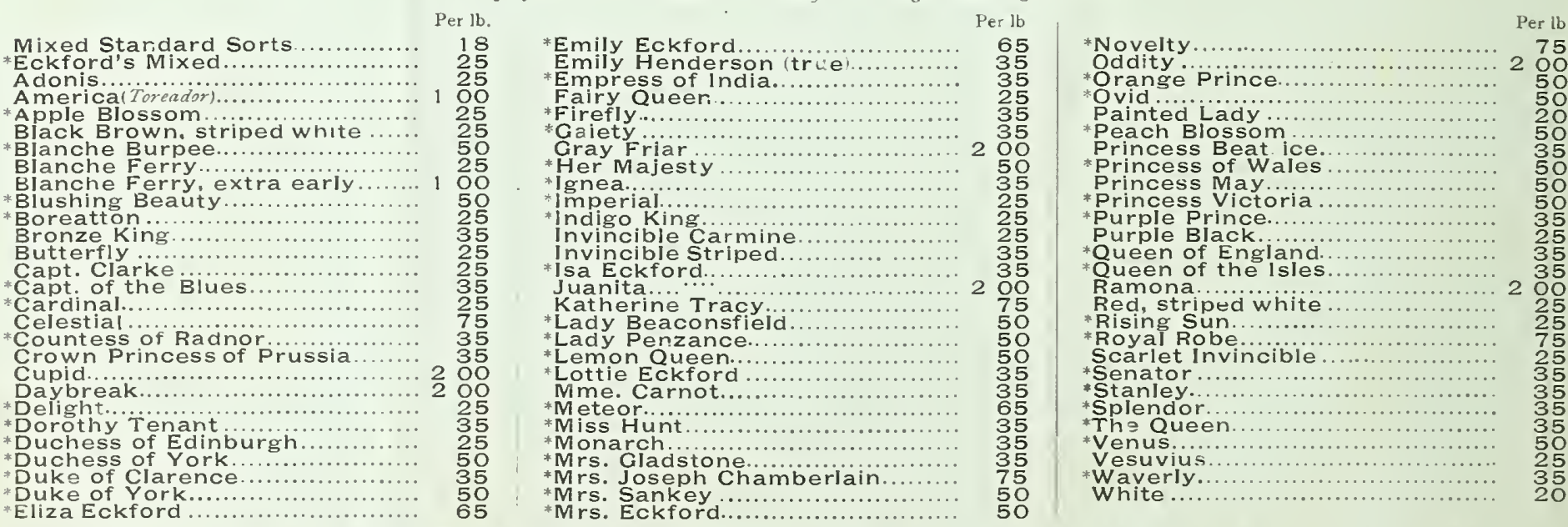

NOTE. - The common and free sceding varieties, blue and purple, do not predominate in our mixtures, but in ours you will find plenty of uright rolors, such as will make your customers so pleased with Sicet Peas that they will come for them again next year.

PETER HENDERSON \& CO., 35 \& 37 Cortlandt St., New York. 

